\title{
IEEE 802.11e-EDCF Evaluation through MAC-Layer Metrics over QoS-aware Mobility Constraints
}

\author{
Khaled Dridi \\ Laboratory of Images, \\ Signals and Intelligent \\ Systems, University of \\ Paris East, France. \\ khaled.dridi@etu.univ-paris12.fr
}

\author{
Nadeem Javaid \\ Laboratory of Images, \\ Signals and Intelligent \\ Systems, University of \\ Paris East, France. \\ nadeemjavaid@ieee.org
}

\author{
Boubaker Daachi \\ Laboratory of Images, \\ Signals and Intelligent \\ Systems, University of \\ Paris East, France. \\ daachi@univ-paris12.fr
}

\author{
Karim Djouani \\ LISSI, University of Paris \\ East, France. \\ djouani@univ-paris12.fr \\ F'SATI, Pretoria, S. Africa. \\ djouanik@tut.ac.za
}

\begin{abstract}
This paper presents how enhanced Quality of Service (QoS) in IEEE 802.11e is achieved by providing traffics with different priorities performing the access to the wireless medium. Particularly, the EDCF set of parameters defines the priorities of the admission control mechanism during the Contention-based Period (CP). This can subsequently be declined to a variation of network dynamicity. Reliability analysis of different traffic classes (video, voice and data), without considering both network topology and node's mobility constraints, is not well appropriate. Being based upon scenarios, our proposed approach reveals how the behaviour of the service differentiation scheme is greatly affected according to the nodes' mobility (position and velocity). Thus, three levels (Low, Medium, and High) of node's speed are discerned. Depending on the Access Aategories (AC's) in QoS Stations (QSAT's), the Wireless LAN has been implemented on various static and dynamic scenarios using NS-2. Performance of EDCF based on the main MAC-layer metrics, such as throughput, End-2-End delay and jitter, is deeply investigated.
\end{abstract}

\section{Keywords}

IEEE 802.11e, EDCF, Mobility, QoS, Throughput, End-2-end delay, Jitter.

\section{INTRODUCTION}

Though wireless networks suffer from lower bandwidth (rather shared bandwidth), higher Bit Error Rate (BER), important amount of delays, and lower security than wired networks, still they have been emerged as a prevailing technology for the broadband wireless access, like IEEE 802.11 WLAN. 'Shared Medium' is the basic cause for the problem which must: provide service differentiation between different types of traffic, risk of collisions, low medium utilization, etc.

Permission to make digital or hard copies of all or part of this work for personal or classroom use is granted without fee provided that copies are not made or distributed for profit or commercial advantage and that copies bear this notice and the full citation on the first page. To copy otherwise, or republish, to post on servers or to redistribute to lists, requires prior specific permission and/or a fee.

MoMM2009, December 14-16, 2009, Kuala Lumpur, Malaysia. Copyright 2009 ACM 978-1-60558-659-5/09/0012...\$10.00.
Moreover wireless networks are much better than the wired networks because of: more flexibility, easy installation, increased bandwidth and very less prices [6]. If the recent standards of IEEE $802.11 \mathrm{x}$ provide the guarantee for connectivity, sufficient coverage, least price, required security and more compatibility among the existing technologies then it is likely that the demands to support real-time applications such as streaming of voice, video and data supported over these networks will increase day-by-day. Particularly, the EDCF set of parameters defines the priorities of the admission control mechanism during the contention-based period (CP). This can subsequently be declined to a variation of network dynamicity. Reliability analysis of different traffic classes (video, voice and data), without considering both network topology and node's mobility constraints, is not well appropriate.

The organization of the paper is as follows:

Section II first describes the background of IEEE 802.11 standard with service differentiation. Then there is a discussion that how IEEE802.11e (EDCF) is evolved from DCF. The section also elaborates the needs of mobility in recent era. Section III is comprised of a detailed discussion of three MAC-layer metrics on which this work has been done. Sections IV gives the description of the topology and scenarios which, we think, are the best suitable for our proposed work. Conclusion is given in section $\mathrm{V}$ and references are provided in section VI. In our last work [1], we proposed a scheduling algorithm aiming to improve $\mathrm{QoS}$ in the wireless networks.

\section{BACKGROUND}

The service differentiation is one of the most wanted demands for the recent applications (with voice, video and data). So, IEEE 802.11 provided a mode of operation for the said demand. But it shows very poor performance regarding link utilization [2]. Several new mechanisms for service differentiation have been and being proposed [3], [4], [5]. The quality of traffics including video streaming is getting better performance because of being considered according to the characteristics of wireless networks and different sorts of service differentiation.

\subsection{Legacy IEEE 802.11 MAC}

The standard WLAN IEEE 802.11 used Best-effort service model built on FIFO queuing mechanism. The access mode is based 
upon two different access methods; the mandatory Distributed Coordination Function (DCF) operates in Contention Period (CP) and Point Coordination Function (PCF) for the polling during Contention Free Period (CFP).

\subsection{DCF}

Carrier Sense Multiple Access with Collision Avoidance (CSMA/CA) protocol is used to regulate the access of the shared medium; the most important dilemma of wireless networks. As discussed above, all wireless nodes have simultaneous access to the shared medium. If a node wants to transmit, it starts to sense the medium state. The frame is transmitted only if the medium is idle for at least a DCF interframe space (DIFS) period of time. If medium is busy, the node chooses a backoff time slot, B, consisting of a random interval 0 to $\mathrm{W}$ (Contention Window). Each time B is decremented by one, when the medium is detected idle for at least a DIFS. Now, during a B if the medium is occupied, the B timer is paused. It is restarted when the medium becomes free for a DIFS in the second time. The frame is transmitted when the backoff timer reaches zero.

If a node does not receive an acknowledgement frame, it is considered that a collision has occurred and the contention window, $\mathrm{W}$, is doubled, as:

$$
W_{n}=2^{c+n-1}-1
$$

Where $n$ is the number of transmission attempts along with the current one for the frame. $c$ is a constant, which defines the minimum contention window, as:

$$
c=\log _{2}\left(W_{\min }+1\right)
$$

To start a backoff procedure, a new allocated time is chosen. Therefore, this mechanism is activated before sending a new frame and/or after the successful transmission of a frame. When a transmission is successful, the contention window is reset to $W_{\min }$.

\subsection{PCF}

This function is related to the existence of a base station. This station performs as Point Coordinator (PC). So, it can be said rightly that $\mathrm{PCF}$ is a centralized and polling-based access mechanism. The condition for the coexistence of both DCF and PCF is the support of PCF. Then each time slot is divided into a superframe, which has two periods: Contention Period (CP) and Contention Free Period (CFP). PCF works in CFP and DCF works in CP. To start a CFP, the base station transmits a beacon frame. Once CFP is started, PC maintains a list of the nodes which have demanded to be polled in order to transmit data, and it sends poll frames to the nodes accordingly [7]. On response, the nodes transmit data packets (if they have in the buffer/queue). A Shorter IFS, between PCF data frames is introduced to avoid the interruption by DCF nodes.

\subsection{IEEE 802.11e-EDCF Mechanism}

To make the existing IEEE 802.11 coordination function more efficient, EDCF mechanism has been proposed, which aims to enhance the access by providing the distributed access for the service differentiation. Task group E have recently proposed this functionality as an extension to the IEEE 802.11 standard, called IEEE 802.11e. The goal is to enhance the access mechanisms and achieve the desired service differentiation level while the main functionality for both protocols remains the same since a lot of devices have been deployed with the old standard. For this reason, the EDCF must follow the backward-compatibility.

The standard 802.11e classifies the traffic into four ACs based on eight user priority levels. Each class has different $W_{\min }$ and interframe space for the transmission of data. If a node, for example, requires higher priority for data transmission, it would be having smaller $W_{\min }$ and hence shorter backoff. If more nodes have the same $W$, the traffic classes are differentiated by having diverse interframe spaces. To avoid waiting a DIFS before accessing the medium or like DCF to decrement backoff timer, a new interframe space called as Arbitration Interframe Space (AIFS) is introduced. As mentioned above that EDCF has eight traffic classes with different AIFS but same DIFS. Fig. 1 demonstrates access mechanisms in EDCF and DCF.
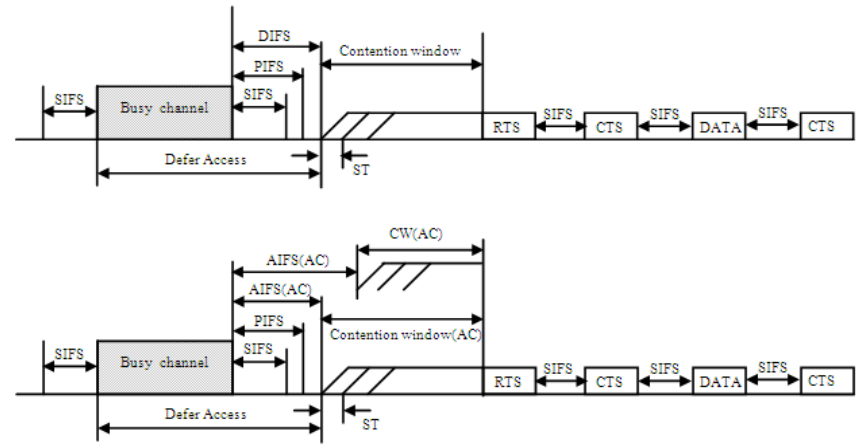

Fig. 1. DCF vs. EDCF access mechanisms.

\subsection{Quality of Service (QoS)}

Quality of Service is considered as a mechanism which aims to satisfy a certain level of service performance within a network. It can also be defined as the ability of an element of network to provide desired handling of traffic according to specific application requirements.

\subsubsection{QOS Limitations in IEEE 802.11 Standards}

If a network supports a set of traffic specifications, as, bandwidth, transmission delay, jitter bound and data path-loss, then it is said to support QoS delivery. It treats packets of all traffic categories at the same level and no service differentiation at all.

Finally, all sorts of traffics, including, voice and video traffics, suffer from delays and bandwidth variations.

\subsubsection{DCF and $Q o S$}

DCF presents "Best-effort Service" model, in which there is no guarantee for throughput, delay and packets-loss. On the other hand, we can simply say that there is no 'service differentiation'.

\subsubsection{PCF and $Q o S$}

PCF mechanism itself weakens QoS; firstly, IEEE802.11a creates a delay of $4.9 \mathrm{~ms}$ because PCF uses beacon frames to separate CP and PCF. Secondly, "all types of traffics are to pass through AP" condition causes decrease in bandwidth. Thirdly, Mac Service Data Unit (MSDU) size is affected by the transmission of data in different sizes, which makes QoS uncertain for remaining CFP period. 


\subsection{Mobility}

With wireless imbedded devices and high requirement for spreading data transferring, offer facilities for several applications aiming to investigate and controlling wide spaces with optimal network architecture, cellular network, sensor network, PDA and smart phones streaming applications as videoconferencing and VOIP constrained by mobility, embedded systems in ground transport (during walk, on cycle, on car, etc) can disturb communication systems which can't follow the small devices. The increase of more requirement for internet keeping high level of QoS and security, make the wireless network as a big challenge. That's why we proposed to evaluate the most spreading network as IEEE802.11 with QoS under the constraint of mobility. For this, the EDCF QoS mechanism is analyzed through the traffic requirements.

To avoid several outside varying parameters according to the fact of mobility, we adopted a scenario with fixed topology and a mobile node as a user connected to two BS's: passes through, disconnect from it, check if it is in the coverage with another BS, connect with it and continue communication according to the handover process.

Depending on the node's velocity, the direction of the motion and the EDCF behavior in QoS stability region, we suggested three ranges of node's velocity: Low [5-12] m/s, Medium [15-30] m/s, and High [40-60] m/s. Exceeding these ranges, the EDCF will be unsteady and the QoS will not be guaranteed.

In the case when the ranges of two BS's overlap the mobile node, the "Multi-coverage Algorithm" is used by the mobile node. This algorithm is given as follows.

\subsection{Multi-coverage Algorithm}

$$
\begin{array}{ll}
1 & m_{n}(x, y) \leftarrow \text { mobile node } \\
2 & b_{s_{1}}\left(x_{1}, y_{1}\right) \leftarrow \text { base station } 1 \\
3 & b_{s_{2}}\left(x_{2}, y_{2}\right) \leftarrow \text { base station } 2 \\
4 & \check{\mathrm{R}}_{1} \leftarrow \text { range of } b_{s_{1}} \\
5 & \check{\mathrm{R}}_{2} \leftarrow \text { range of } b_{s_{2}} \\
6 & d_{1} \leftarrow\left|m_{n} b_{s_{1}}\right| / / \text { distance between } m_{n} \text { and } b_{s_{1}} \\
7 & d_{2} \leftarrow\left|m_{n} b_{s_{2}}\right| / / \text { distance between } m_{n} \text { and } b_{s_{2}} \\
8 & d_{1} \leftarrow \sqrt{\left(x-x_{1}\right)^{2}+\left(y-y_{1}\right)^{2}} \\
9 & d_{2} \leftarrow \sqrt{\left(x-x_{2}\right)^{2}+\left(y-y_{2}\right)^{2}} \\
10 & \text { for }\left\{m_{n}(x, y) \subset\left(\check{\mathrm{R}}_{1} \cap \check{\mathrm{R}}_{2}\right)\right\} \\
11 & \text { if }\left(d_{1}<=d_{2}\right) \\
12 & \left\lfloor\text { then connect } m_{n} \text { to } b_{s_{1}}\right. \\
13 & \text { else } \\
14 & \text { connect } m_{n} \text { to } b_{s_{2}} \\
15 & \text { for }\left\{m_{n}(x, y) \not \subset\left(\check{\mathrm{R}}_{1} \cap \check{\mathrm{R}}_{2}\right)\right\} \\
16 & \text { if } \left.\left\{m_{n}(x, y) \subset \check{\mathrm{R}}_{1}\right)\right\} \\
17 & \text { then connect } m_{n} \text { to } b_{s_{1}} \\
18 & \text { else }
\end{array}
$$

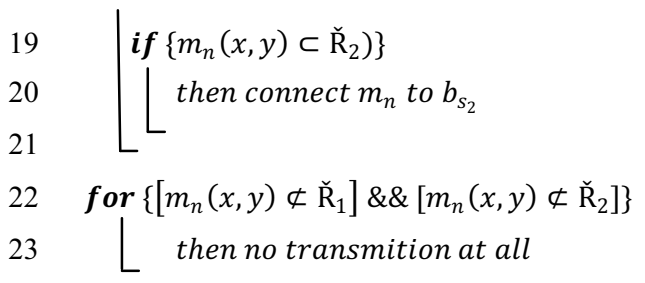

\section{MAC LAYER METRICS}

A metric is a value assigned to each link (path or route) to be used by an algorithm or a protocol. It aims to choose the best one out from an available lot of links for data transmission. As we have seen that wireless networks have shared bandwidth and to make sure that the limited resources of a wireless network be used efficiently, the paths selected by the protocol must have good performance to achieve high throughput network.

Results of simulations allow to see the behavior of EDCF in different mobility levels with three MAC-layer metrics: throughput, end-2-end delay and jitter, it is worth discussing these metrics first.

\subsection{Effective Throughput}

The amount of data in (bits or bytes) successfully transferred from source to destination or it can be measured on hop-to-hop basis, is called throughput. This metric operates as a gauge to measure the amount of data successfully transferred from hop/source to hop/destination in a particular period of time. Bits per second $(\mathrm{b} / \mathrm{s})$, bytes per second $(\mathrm{B} / \mathrm{s})$, frames per second $(\mathrm{f} / \mathrm{s})$, and symbols per second (s/s) are the typical measuring units of throughput.

$$
\text { Throughput }=\frac{\sum_{n} \text { Received Packets }}{\sum_{t} \text { Time }} \quad B / s
$$

To be well estimated this metric, only received packets are considered in our computation.

\subsection{End-2-End Delay (E2ED)}

E2ED is the time a packet takes to reach the destination from the source. E2ED or latency can rightly be considered as propagation delay from the source to the destination without any extra processing involved, as, packetization delay at the generator or the packet's analyzer at the destination.

$$
\text { E2ED }=\text { Arrival Time }- \text { Departure Time } \quad s
$$

There also exists the possibility to measure E2ED delay as the mean of round trip time.

$$
E 2 E D_{\text {avg }}=\frac{\sum_{n} R T T_{n}}{n} \quad S
$$

\subsection{Jitter formulas}

Variation in the E2ED measured at the destination is called jitter. The time-sensitive applications, as video or voice streaming, are affected due to the high variations in the jitter. This metric can well estimate and evaluate the performance of the traffic-sensitive applications.

Depending upon the requirements, there are numerous ways to measure the jitter. 


\subsubsection{Jitter combined with E2ED}

Here, jitter is measured by calculating the difference in the maximum and minimum E2ED. Most of the equipments deployed to-date provide maximum and minimum E2ED measurement, because this is the easiest and convenient method for measuring jitter.

$$
\text { Jitter }=\text { Max E2ED }- \text { Min E2ED } \quad s
$$

\subsubsection{Jitter relating to arrival time of packets}

Here, jitter is measured by measuring the difference in the arrival time of the packets at the destination instead of E2ED.

$$
\delta a_{n}=a_{n}-a_{n-1} \quad s
$$

where, $n$ shows the current packet, $a_{n}$ is the arrival time of the current packet and $a_{n-1}$ is the arrival time of the previous packet.

$$
\text { Jitter }_{n}=\delta a_{n}-\delta a_{n-1} \quad s
$$

\subsubsection{Jitter relating to consecutive packets}

Here, jitter is measured by measuring the difference in the E2ED of current packet and the previous packet [8].

$$
\text { Jitter }_{n}=E 2 E D_{n}-E 2 E D_{n-1} \quad s
$$

E2ED can also be expressed as:

$$
E 2 E D_{n}=a_{n}-d_{n} \quad s
$$

Here $E 2 E D_{n}$ is always positive, as $a_{n}>d_{n}$.

Now, jitter from Eq.(4) can be rewritten as:

Then jitter can be written as,

$$
\begin{array}{cc}
\text { Jitter }_{n}=\left(a_{n}-d_{n}\right)-\left(a_{n-1}-d_{n-1}\right) & s \\
\text { Jitter }_{n}=\left(a_{n}-a_{n-1}\right)-\left(d_{n}-d_{n-1}\right) & s \\
\text { Jitter }_{n}=\delta a_{n}-\delta d_{n} & s
\end{array}
$$

If we look closely, the jitter measured in method 3 in Eq. (9) is similar to method 2 in Eq. (6). $\delta d_{n}$ is an addition in Eq. (9), which obviously shows the difference in the departure times. If the generator at the source is sending the packets at variable rate, the term $\delta d_{n}$ will compensate this error.

\subsubsection{Jitter relating to current and average E2ED}

Here, jitter is measured by computing the difference in the E2ED of $n^{\text {th }}$ packet. It is worth noting that average E2ED is measured over the required duration of analysis.

$$
\operatorname{Jitter}_{n}=E 2 E D_{n}-E 2 E D_{a v} \quad s
$$

\section{SCENARIOS}

We started to analyze the impact of the network variations topology and dynamicity on the stability of EDCF mechanism using NS-2 [10]. Results of the comparison study between static scenarios, took as a reference and dynamic scenario show the degree of sensitivity of QoS service delivery to the mobile environment. The degradation rate is important where the EDCF can reach the instability region. Consequently, it loses significantly its service differentiation quality and looks like DCF in the worst case of simulation, as shown in Fig. 1 and Fig. 2. The present study focuses on the stability region of EDCF under mobility constraint. QoS scheme is evaluated depending on three domains of velocity (Low, Medium, and High) through the set of MAC-layer metrics. We proposed a hybrid network, which is composed of wireless and wired nodes, which can communicate through Base Stations (BS's, $b_{s_{1}} \& b_{s_{2}}$ ). We specified a solution with two BS's observe that what happens when a Mobile Node $\left(m_{n}\right)$ moves out from one BS and enters in the range of another.

At this time, EDCF mobility behaviour can be well evaluated. For the different Access Categories (AC's) of service and to avoid TCP control packets exchanges', 4 CBR-traffics based on UDP transmission protocol are used. To focus only on the impact of mobility, the present study doesn't take care of the fading in the wireless channel and the non-stationary flows in high data rate. 5 Mbps are supported by the wired links, and 1 Mbps of data-rate in wireless medium is shared by the different classes of traffic. As our work focuses MAC layer, so, without making any comparison of network layer protocols, we used Destination Sequenced Distance Vector (DSDV) as routing protocol. To appear congestion event, we preferred limiting at 50 packets the maximum size of the queues, allowing by this dropped packets generation, as in a real network, when the scheduler is out of its capacity.

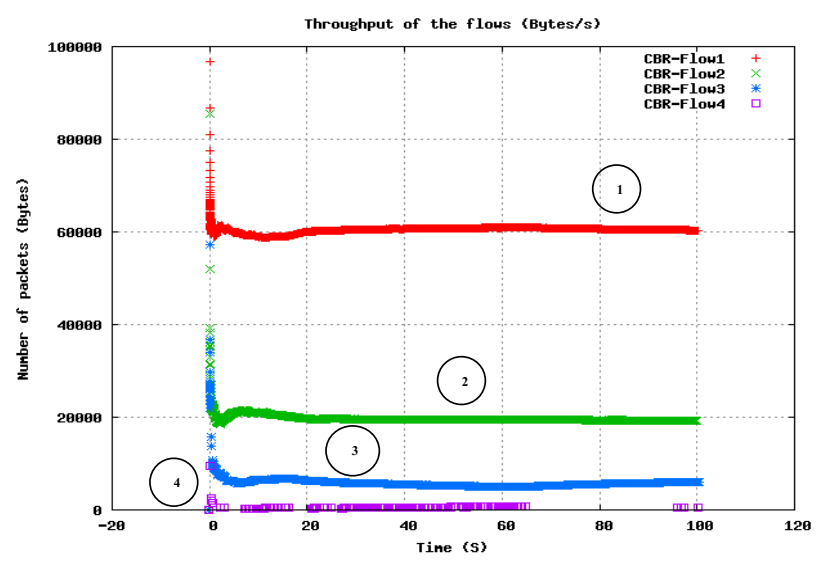

Fig. 2. Throughput without mobility

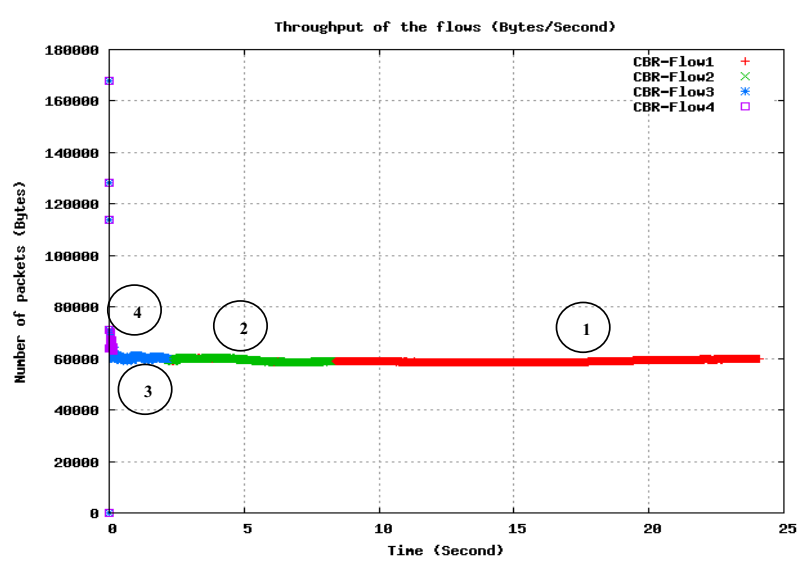

Fig. 3. Throughput with mobility 


\subsection{Low mobility}

\subsubsection{Throughput}

During low mobility and the connection with the BS1, throughput achieves levels $60 \mathrm{kBps}$ and $20 \mathrm{kBps}$ for $\mathrm{AC0}$ and $\mathrm{AC} 1$ respectively. These tow traffics share $80 \%$ of total bandwidth, as depicted in Fig. 4.

During connection with the BS2, the traffic switches into the half level of the top start throughputs $(30 \mathrm{kBps} \& 10 \mathrm{kBps})$ and increases during the range of mobility. In this class of mobility, there is no rapid saturation event and the EDCF has enough gap to track the mobility by arising the throughput application level adequately. By no need of high throughput network resource, VOIP applications can be well transmitted without be thresholded by saturation. QoS is maintained and the behaviours remain the same in both AC's traffic categories with slight rapidness has been expected in $\mathrm{AC} 1$ to reach the top (in border of $12 \mathrm{~m} / \mathrm{s}$ ).

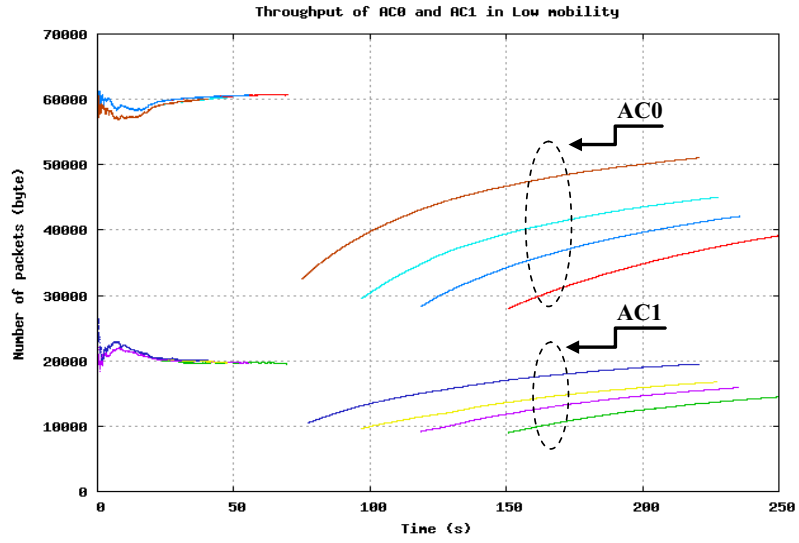

Fig. 4. Throughput for low mobility.

\subsubsection{E2ED}

During the connection with BS2, the AC0 stays around $0.2 \mathrm{~s}$, which is very convenient for real-time and almost delaydependent applications, like audio and video streaming. Unfortunately, EDCF shows some efficiency for AC0 class only. On the other hand, during connection with $\mathrm{BS} 2$ in $\mathrm{AC} 1, \mathrm{EDCF}$ shows some weaknesses and is still sensitive under mobility. As depicted in the graph in Fig. 5, level of $0.8 \mathrm{~s}$ is reached for several times. The limit of $0.2 \mathrm{~s}$ for the reasonable VOIP conversation is exceeded. Fortunately, the value of $0.5 \mathrm{~s}$ is rapidly bounded which can be welcomed for CBR-MPEG video stream applications.

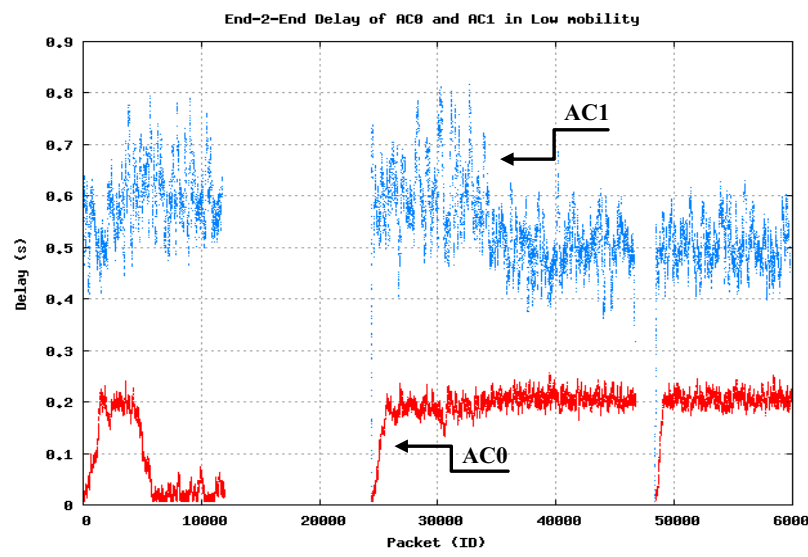

Fig. 5. E2ED for low mobility.

\subsubsection{Jitter}

The graph in the Fig. 6 shows the jitter plotted in two sides of $x$ axis. Referring to each previous transmission, the packets arrive early are represented by the negative peaks and positive peaks show the packets which arrive late.

The variation of intensity in both sides identifies the quality of transmission (e.g. degradation quality during a call in VOIP).

For AC0, the jitter is bounded around $0.01 \mathrm{~s}$ for the all transmissions. An interesting behaviour of EDCF is observed at the negative side. The flow stays quite stable before and after the switching period between the BS's. It shows the ability of the EDCF scheduler to track jitter measurements and adjust buffer size to reduce the jitter impacts.

Unfortunately, as we can see in the same graph, this behaviour raises the jitter impact of $\mathrm{AC} 1$ (it can reach $0.06 \mathrm{~s}$ with $0.12 \mathrm{~s}$ peakto-peak of variance). Far from this region, EDCF with CBR flow is quite steady.

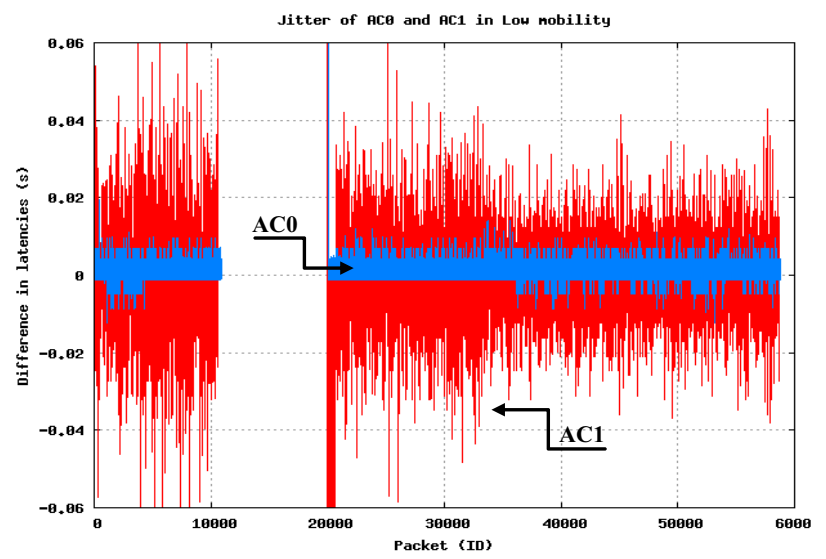

Fig. 6. Jitter for low mobility.

\subsection{Medium Mobility}

\subsubsection{Throughput}

At the start, during connection with $\mathrm{BS} 1$, the top $\mathrm{AC} 0$ and $\mathrm{AC} 1$ throughputs stay unchanged. In AC0 traffics, with the increase of the node's mobility, the throughput slightly increases. Comparing to the previous mode, the graph for this case (Fig. 7) is much closer to the previous case (Fig. 4). The throughput can't grow over $57 \mathrm{kBps}$ (start of saturation bound). Even if the start top level is not reached; EDCF is still not able to increase the throughput for the highest priority traffics. In contrast to $\mathrm{AC} 0, \mathrm{AC} 1$ can gain more flexibility and it can increase up to the top start level even the saturation in curves is expected (more than $20 \mathrm{kBps}$ reached in $30 \mathrm{~m} / \mathrm{s})$.

In this mobility mode, EDCF guarantees maximum throughput. This is highly required variation in throughput for the sensitive bit rate application (VBR flow) [8], as they need robustness over user mobility. Comparing the connections with the BS's in both cases, we can observe that $\mathrm{AC} 1$ does not waste as much bandwidth, as AC0 does. 


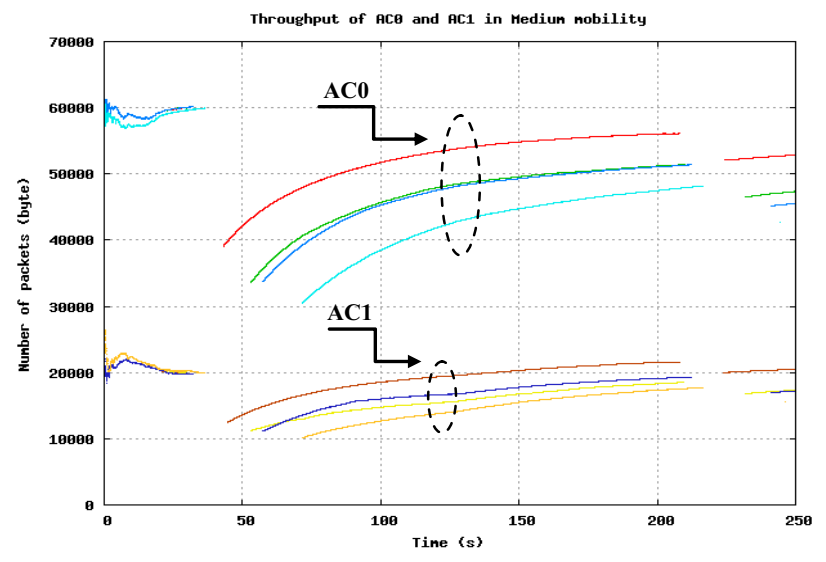

Fig. 7. Throughput for medium mobility.

\subsubsection{E2ED}

AC0 E2ED gains more stability after connection with BS2 down to $0.1 \mathrm{~s}$, and up at $\sim 0.2 \mathrm{~s}$. This zone can be reserved for high sensitive traffics for small period of time (as in Burst mode). The AC0 in the graph depicted in Fig. 8, shows that around 30000 packets are allowed for one burst. AC1, after establishing the connection $(0.5 \mathrm{~s})$ attains an average delay and stays steady for the rest of transmission.

This is the most important feature of this mode that in the same transmission, it can bring to high priority traffic with a small shift of delay but guaranteeing a maximum stability. We find the best result at $30 \mathrm{~m} / \mathrm{s}$.

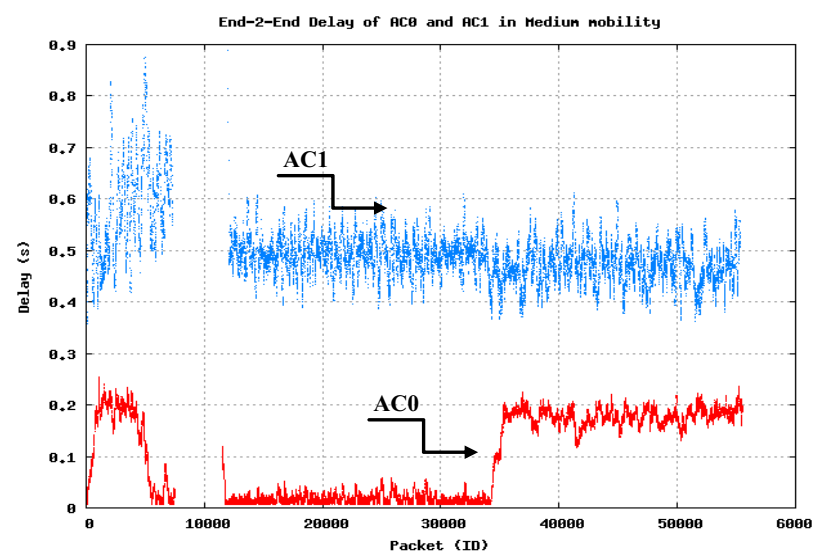

Fig. 8. E2ED for medium mobility.

\subsubsection{Jitter}

As shown by Fig. 9, high stability of the negative side of AC0 (before the range of 35000 packet ID) shows that no packets are coming early. AC1 proves E2ED stability with a reasonable level of jitter $(<0.5 \mathrm{~s})$ after connection with BS2, with $0.1 \mathrm{~s}$ peak-to-peak of variance. Almost all of the traffics can support the range of mobility of this mode with a condition of burst (as explained previously).

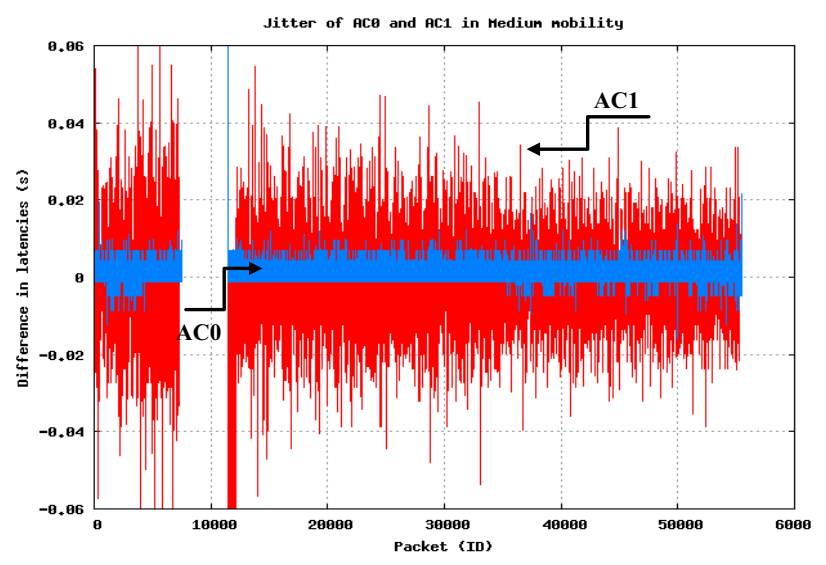

Fig. 9. Jitter for medium mobility.

\subsection{High Mobility}

\subsubsection{Throughput}

Within this mode, $\mathrm{AC} 0$ and $\mathrm{AC} 1$ keep the same behaviours independently of the node's mobility, as shown in Fig. 10. EDCF lasts a throughput level comparing to the other modes $(15 \mathrm{~Kb} / \mathrm{s}$, $30 \mathrm{~Kb} / \mathrm{s}$ for $\mathrm{AC} 0$ and $\mathrm{AC} 1$ respectively). It cannot track the varying motion to adjust the throughput accordingly. This behaviour is mainly involved to the size of buffer of the scheduler which is not able to support higher speeds $(40 \mathrm{~m} / \mathrm{s}$ as a critical). All of the traffic classes are penalized with a fixed level of throughput depending on the configured buffer parameters (size of queue and queuing scheduling strategy) [9].

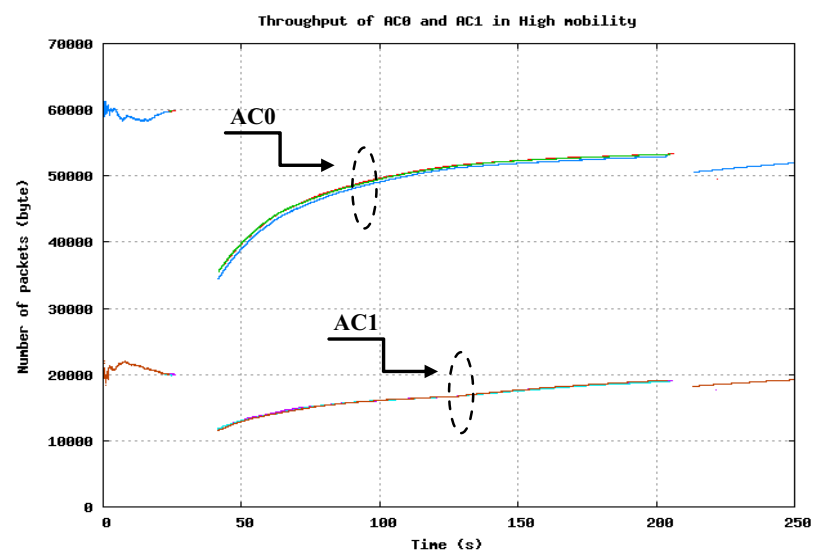

Fig. 10. Throughput for high mobility.

\subsubsection{E2ED}

AC0 stays in fixed position $(0.2 \mathrm{~s})$ as the previous mode. AC1 shifted with high delay $(0.9 \mathrm{~s})$ and stays in the same level independently of the mobility in the network.

The Fig. 11 shows the stability of AC0 and high level of delay by which $\mathrm{AC} 1$ is affected. 


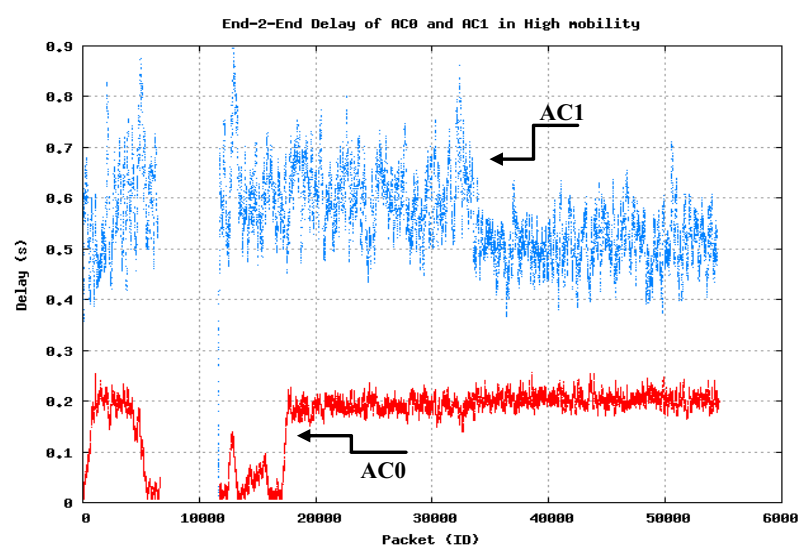

Fig. 11. E2ED for high mobility.

\subsubsection{Jitter}

$\mathrm{AC} 0$ is declined more and more and reached worst level comparing to the medium mode, as stability is decreased (less than 10000 packets for burst). AC1 keeps the same levels of jitter ( $0.06 \mathrm{~s}$ with 0.12 peak-to-peak of variance in the spectrum of the Fig. 12) with random concentration slightly appeared and can be discerned in the path of mobility.

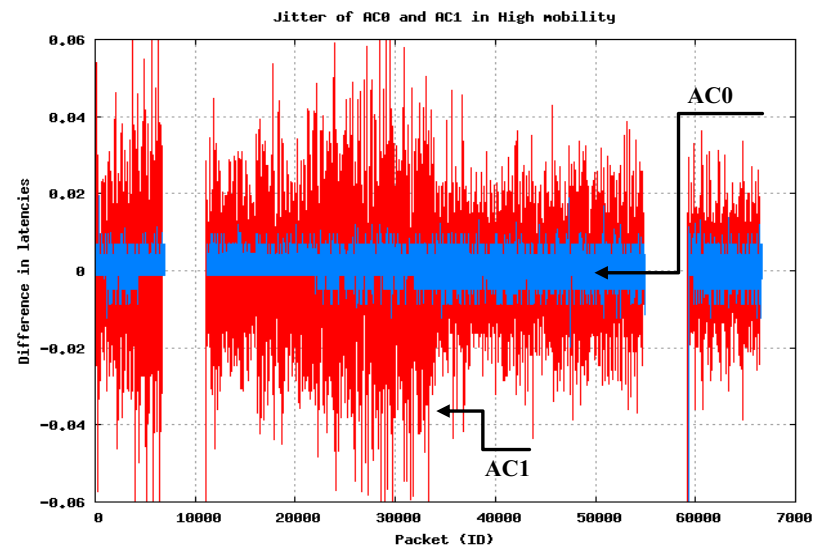

Fig. 12. Jitter for high mobility.

Table. 1. summarizes the behaviour of EDCF under the three mobility domains.

\begin{tabular}{|c|c|c|c|}
\hline Mobility & Low & Medium & High \\
\hline Throughput & $\begin{array}{c}\text { Best } \\
\text { for real-time } \\
\text { applications } \\
\text { \& video } \\
\text { conferencing }\end{array}$ & $\begin{array}{c}\text { Satisfactory } \\
\text { for Data } \\
\text { transmission }\end{array}$ & $\begin{array}{c}\text { Satisfactory } \\
\text { for emails, } \\
\text { SMS, MMS }\end{array}$ \\
\hline E2ED & $\begin{array}{c}\text { Good for all } \\
\text { streaming } \\
\text { traffics }\end{array}$ & $\begin{array}{c}\text { Satisfactory } \\
\text { for CBR- } \\
\text { MPEG }\end{array}$ & $\begin{array}{c}\text { Not } \\
\text { satisfactory } \\
\text { for all traffics }\end{array}$ \\
\hline Jitter & $\begin{array}{c}\text { Good } \\
\text { for VOIP } \\
\text { traffic }\end{array}$ & $\begin{array}{c}\text { Good } \\
\text { for non- } \\
\text { streaming } \\
\text { traffics }\end{array}$ & $\begin{array}{c}\text { Worst } \\
\text { Critical level } \\
\text { of packet drop }\end{array}$ \\
\hline
\end{tabular}

Table. 1. Behavior of EDCF with different Mobilities

\section{CONCLUSION AND FUTURE WORK}

In our study, we focused on the performance of the EDCF protocol under the node's mobility constrains. The IEEE802.11 reveals high sensitivity to the nodes' position and velocity. These can significantly decrease the standard service ability. We focus on looking for stability region of the MAC protocol, several tests are performed over the main layer-metrics; throughput, End-2End delay and jitter in order to quantify this impact and relating it to different classes of traffic. We ended by proposing a benchmark which summarized the impact of these metrics according to three zones of stability. Therefore, depending on network scenario implemented in NS-2 simulations, the QoS mechanism behaved differently. The study of MAC protocol, even the range is limited by PHY layer comparing to the IEEE802.16 standard, allows extension, since it can operate easily within cooperation topology. The approved results can help users to identify the borderline of service's steadiness depending on the requirements of the traffic. Following this optimistic approach, we plan to enhance this study by supporting channel fading effect, multipath interferences and link adaptation quality within node's cooperation diversity network.

\section{REFERENCES}

[1] K. Dridi, K. Djouani and B. Daachi, "Three Schedulinglevels algorithm for IEEE 802.11e QoS efficiency improvement", 6th International Conference on Advances in Mobile Computing \& Multimedia (MoMM), 2008.

[2] M.A. Visser and M. El Zarki, Voice and data transmission over an 802.11 wireless network, in: Proceedings of PIMRC'95, Toronto, Canada (1995) pp. 648-652.

[3] Dongxia Xu, Taka Sakurai, and Hai L. Vu, An Access Delay Model for IEEE 802.11e EDCA, IEEE TRANSACTIONS ON MOBILE COMPUTING, VOL. 8, NO. 2, Feb, 2009.

[4] I-Shyan Hwang, Jheng-Han Wu, "Performance assessment of service differentiation in IEEE 802.11e Wireless LANs", International Journal of Ad Hoc and Ubiquitous Computing 2008 - Vol. 3, No.1 pp. 21 - 32.

[5] Whe-Dar and Der-Jiunn Deng, "Service Differentiation in IEEE 802.11e HCF Access Method", Springer Berlin / Heidelberg, 2008.

[6] IEEE K. Std. 802.11-1999, Part 11: Wireless LAN Medium

Access Control (MAC) and Physical Layer (PHY) specifications, Reference number ISO/IEC 8802-11:1999(E), IEEE Std. 802.11, 1999 edition, 1999.

[7] Tehuang Liu, Wanjiun Liao, and Jeng-Farn Lee, "Distributed Contention-Aware Call Admission Control for IEEE 802.11 Multi-Radio Multi-Rate Multi-Channel Wireless Mesh Networks," ACM/Springer, 2009.

[8] Rong He; Xuming Fang, "A fair MAC scheme for EDCA based wireless networks", Testbeds and Research Infrastructures for the Development of Networks \& Communities and Workshops, 2009.

[9] Rong He; Xuming Fang, "A Fair MAC Algorithm with Dynamic Priority for 802.11e WLANs”, ICCSN, 2009.

[10] Technical Report TKN-06-003, S.Wiethölter, M.Emmelma., C.Hoene, A.Wolisz "TKN EDCA Model for ns-2", Technische Universität Berlin, June 2006. 